\title{
BMJ Open How to train practising gynaecologists in total laparoscopic hysterectomy: protocol for the stepped-wedge IMAGINE trial
}

\author{
Andreas Obermair, ${ }^{\oplus 1,2}$ Nigel R Armfield, ${ }^{\odot 1}$ Nicholas Graves, ${ }^{\odot 3}$ Val Gebski, ${ }^{4}$ \\ George B Hanna, ${ }^{5}$ Mark G Coleman, ${ }^{6}$ Anne Hughes, ${ }^{1}$ Monika Janda ${ }^{\oplus, 8}$
}

To cite: Obermair A, Armfield NR, Graves N, et al. How to train practising gynaecologists in total laparoscopic hysterectomy: protocol for the stepped-wedge IMAGINE trial. BMJ Open 2019;9:e027155. doi:10.1136/ bmjopen-2018-027155

\section{- Prepublication history and} additional material for this paper are available online. To view these files, please visit the journal online (http://dx.doi. org/10.1136/bmjopen-2018027155).

Received 9 October 2018 Revised 8 March 2019 Accepted 12 March 2019
Check for updates

(C) Author(s) (or their employer(s)) 2019. Re-use permitted under CC BY-NC. No commercial re-use. See rights and permissions. Published by BMJ.

For numbered affiliations see end of article.

Correspondence to Professor Andreas Obermair; ao@surgicalperformance.com

\section{ABSTRACT}

Introduction Hysterectomy is the most common major gynaecological procedure in women and minimally invasive approaches should be used wherever possible; total laparoscopic hysterectomy (TLH) is one such surgical approach which allows removal of the uterus entirely laparoscopically. However, lack of surgical training opportunities is impeding its increased adoption. This study will formally test a surgical outreach training model to equip surgeons with the skills to provide TLH as an alternative to total abdominal hysterectomy (TAH). Methods and analysis Stepped wedge implementation trial of a surgical training programme for practising obstetrician gynaecologist specialists in four hospitals. Primary outcomes Change in the proportion of hysterectomies performed by TAH, measured between preintervention and postintervention; we aim to reduce TAH by at least $30 \%$ in $75 \%$ of the trainees.

Secondary outcomes (1) Number of hospitals screened, eligible, agree to training and complete the training; (2) number of surgeons screened for eligibility, eligible, agree to training, who complete training and achieve proficiency; (3) proportion of trainees achieving proficiency in correct theatre setup, vascular exposure, mobilisation and surgery closure; change in proportion proficient over time; (4) adverse events (conversion from TLH to TAH, anaesthetic incident, intraoperative visceral injury, red cell transfusions, hospital stay $>7$ days, incidental finding of malignancy, unplanned readmission, admission to intensive care, return to theatre, postoperative pulmonary embolism or deep vein thrombosis, development of a fistula, vault haematoma, vaginal vault dehiscence or pelvic infection); (5) hospital length-of-stay; (6) cost-effectiveness and (7) trainee surgeon proficiency with TLH.

Ethics and dissemination The study has been approved by the Royal Brisbane and Women's Hospital Human Research Ethics Committee and has received site-specific approval from all participating hospitals. Results will be submitted for publication in a peer-reviewed journal. Trial registration number NCT03617354; Pre-results.

\section{INTRODUCTION}

Hysterectomy is the most common major gynaecological procedure in women. ${ }^{1}{ }^{2}$ Reviews and meta-analyses ${ }^{3-6}$ conclude that

\section{Strengths and limitations of this study}

- The study will be the first to formally assess a structured training programme for obstetrician-gynaecologist specialists in total laparoscopic hysterectomy.

- The primary outcome measure, a reduction in open surgery, is patient-focussed and clinically meaningful.

- The secondary outcome measures will allow the economics of structured training to be assessed from the health service perspective.

- The study is limited by the practicalities of a small number of study sites and trainees; however, it will provide evidence to inform scaling up of training and will allow transferability of educational programmes into new surgical techniques.

minimally invasive approaches should be preferred over total abdominal hysterectomy (TAH), and should be considered whenever clinically possible. ${ }^{3-11}$

The American College of Obstetricians and Gynaecologists (ACOG), American Association of Gynecologic Laparoscopists (AAGL), Society of Gynecologic Oncology (SGO), European Society for Medical Oncology (ESMO) and the Society of Obstetricians and Gynaecologists of Canada (SOGC) have all published guidelines highlighting the benefits of minimally invasive surgery for women with benign and malignant gynaecological conditions. $^{12}$ Despite the evidence base supporting minimally invasive approaches, and the recommendations by professional societies to decrease $\mathrm{TAH}$, almost $40 \%$ of hysterectomies in Australia are still performed using this approach, and a similar proportion of cases are done using a vaginal approach. ${ }^{1}$

Total laparoscopic hysterectomy (TLH) is a minimally invasive surgical approach to remove the uterus, with or without the adnexae, to treat benign gynaecological 
conditions such as uterine fibroids and adenomyosis or to prevent or treat cancers of the cervix, uterus, fallopian tubes or ovaries. TLH was developed to allow the surgery to be completed entirely laparoscopically, ${ }^{13} 14$ and has been shown to be feasible and safe; compared with TAH, TLH is associated with improved recovery, shorter hospital stay, reduced risk of surgical complications $^{3}{ }^{15-18}$ and equivalent disease-free outcomes for treating endometrial cancer. ${ }^{11}{ }^{19-22}$ While TLH is a slightly more costly procedure than TAH, TLH has been shown to be cost-effective when the total cost of care is considered. ${ }^{719}$ Robotic hysterectomy is an alternative approach to hysterectomy used in some highly developed countries, especially the USA. However, its use in other countries, including Australia, is still limited due to the significant costs associated with robotic technology. ${ }^{23-26}$

\section{RATIONALE}

To investigate why TAH was still commonly used, a survey of Australian and New Zealand gynaecologists identified two main barriers impeding the uptake of TLH: (1) surgeons' lack of procedural skills for TLH and (2) the limited availability of structured training and mentoring opportunities to assist practising surgeons to gain those skills. ${ }^{27}$ Women who have had a hysterectomy were surveyed and reported that they commonly follow the advice of their doctor with regards to the type of hysterectomy, and rarely reported to seek a second opinion. ${ }^{28}$

International evidence shows that structured education and training is effective in decreasing the use of TAH; in Finland, between 1996 and 2006, the proportion of hysterectomies conducted by TAH reduced from $58 \%$ to $24 \%$ through training and education. In parallel, postoperative complications also decreased significantly. ${ }^{17} 29$ In Canada, between 2005 and 2012, the proportion of hysterectomies conducted by TLH increased from $40 \%$ to $74 \%$ through stakeholder engagement and structured learning. ${ }^{30}$
In Australia, no other formal training programme exists to teach advanced laparoscopic techniques, including laparoscopy; this study will implement and evaluate a model of training for practising gynaecologists in TLH.

\section{OBJECTIVES}

\section{Primary objective}

To decrease the proportion of hysterectomies conducted by TAH by $30 \%$, in $75 \%$ of gynaecological surgeons through a surgical outreach training programme delivered at the trainee's hospital.

\section{Secondary objectives}

By decreasing the proportion of patients who receive a TAH, reduce:

1. Incidence of surgical adverse events (AEs) in patients receiving a hysterectomy by $20 \%$.

2. Length of stay (LoS) for patients requiring a hysterectomy by $20 \%$.

3. Direct hospital costs for hysterectomy by $10 \%$.

And to:

1. Evaluate surgeon trainees' experiences of the training programme.

2. Assess satisfaction with the training programme, and the views of relevant stakeholders on benefits and barriers to training.

\section{METHODS AND ANALYSIS \\ Study design and setting}

The Implementation of Minimally Invasive Hysterectomy Trial (Imagine) will follow a stepped wedge implementation trial (figure 1) of a surgical training programme for practising obstetrician-gynaecologist specialists in four hospitals, to reduce the proportion of patients who receive a TAH by increasing the proportion who may receive a TLH instead.

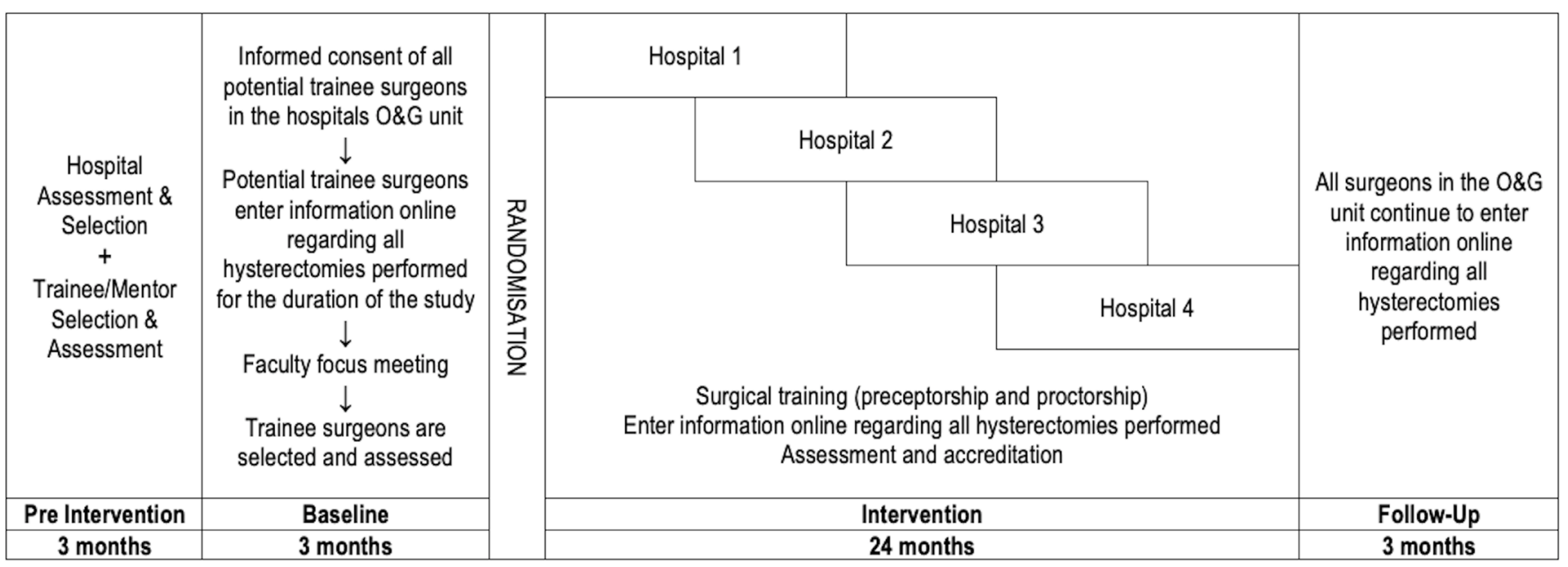

Figure 1 Study schema. 
Table 1 Hospital, mentor and trainee selection criteria

\section{Selection criteria}

\begin{tabular}{|c|c|}
\hline \multicolumn{2}{|l|}{ Training hospital } \\
\hline Institutional support & $\begin{array}{l}\text { Strong desire to change clinical practice in the hospital. } \\
\text { Management support for the further training of its specialists. } \\
\text { Management commitment to STs maintaining the learnt surgical skills following the training } \\
\text { period. } \\
\text { Willingness to adjust rosters so trainees may be available for training. }\end{array}$ \\
\hline Case load & $\begin{array}{l}\text { Sufficient ongoing hysterectomy case load to maintain learnt surgical skills; } \geq 2 \\
\text { hysterectomies/month/ST. }\end{array}$ \\
\hline $\begin{array}{l}\text { Current level of open } \\
\text { surgery }\end{array}$ & $\begin{array}{l}\text { A high proportion (a rate that the hospital considers too high, and wishes to reduce) of } \\
\text { hysterectomies being conducted by TAH. }\end{array}$ \\
\hline Theatre resources & $\begin{array}{l}\text { Willingness and ability to provide a dedicated training operating theatre that is free of the } \\
\text { demands of health service efficiency targets. }\end{array}$ \\
\hline Availability of baseline data & $\begin{array}{l}\text { Readily available information on surgical activity and outcomes at baseline, and any local } \\
\text { barriers to further adoption of TLH. }\end{array}$ \\
\hline Supporting staff & $\begin{array}{l}\text { To ensure that experience and expertise is developed from both a surgical and a nursing } \\
\text { point of view, identification of scrub nurses to be involved in the training process throughout. }\end{array}$ \\
\hline \multicolumn{2}{|l|}{ Surgical mentor } \\
\hline $\begin{array}{l}\text { Laparoscopic hysterectomy } \\
\text { skills and experience }\end{array}$ & $\begin{array}{l}\text { Senior laparoscopic surgeons, proficient in TLH with a track record of } \geq 500 \text { successfully } \\
\text { completed TLHs. }\end{array}$ \\
\hline Working relationships & $\begin{array}{l}\text { To minimise the risk of distracting emotional interference, the SM should have no previous } \\
\text { working or personal relationship with the STs (ie, mentor cannot train their own colleagues). } \\
\text { Willingness to participate in all aspects of the preparation and training at their assigned } \\
\text { hospital. }\end{array}$ \\
\hline
\end{tabular}

\begin{tabular}{|c|c|}
\hline \multicolumn{2}{|l|}{ Surgical trainee } \\
\hline Motivation & Motivated to learn the new surgical technique. \\
\hline $\begin{array}{l}\text { Proficiency in general } \\
\text { laparoscopic surgical } \\
\text { procedures }\end{array}$ & $\begin{array}{l}\text { Such as ectopic pregnancy, laparoscopic ovarian cystectomy or laparoscopic resection of } \\
\text { endometriosis (in the Australian system, RANZCOG accredited O\&G specialists, proficient in } \\
\text { RANZCOG laparoscopic skills level 3), }{ }^{44} \text { with video recordings of surgical skills assessed by } \\
\text { two independent surgeons using an adapted GOALS tool for hysterectomy. }{ }^{45}\end{array}$ \\
\hline Commitment to document & - Commitment to documenting their surgical procedures and outcomes. \\
\hline Availability & $\begin{array}{l}\text { For efficiency and to maximise experiential learning, it should be the aim that all STs are } \\
\text { present on all training days. }\end{array}$ \\
\hline
\end{tabular}

Training case selection
Criteria
Suitable patient classified as low risk, as measured by the SurgicalPerformance Risk of Surgical Complications app (RISC). ${ }^{33}$
- Uterus size $<10$ weeks.
- No previous laparotomy.
- $\leq 2$ previous caesarean sections.
- A reasonably mobile uterus.
- Not being on blood thinning medication.

RANZCOG, Royal Australian and New Zealand College of Obstetricians and Gynaecologists; SM, surgical mentors; ST, surgical trainees; $\mathrm{TAH}$, total abdominal hysterectomy; TLH, total laparoscopic hysterectomy.

\section{Study population}

The participants in this study include the trainer surgeons and trainee surgeons, theatre staff and hospital administrators.

\section{Inclusion criteria}

Inclusion criteria are provided in table 1.

Hospitals are eligible if there is institutional support and perceived need to increase the uptake of TLH, if there are at least two potentially eligible surgeons willing to be trained, if sufficient cases are available at the hospital for training and maintenance of benefit after training ceases and a training operating theatre exempt from national elective surgery targets can be made available. Hospitals can express desire to participate or be approached by the study team.

Surgical mentors (SM) must be experienced in both TAH and TLH, have completed $\geq 100 \mathrm{TLH}$ procedures, have no personal or professional relationship with the 
trainee surgeons that would impede training, have completed a train-the-trainer course and be willing to provide the necessary teaching and support for trainees and complete all relevant assessment and reporting.

Surgical trainees (ST) must be Fellows of the Royal Australian and New Zealand College of Obstetrics and Gynaecology (RANZCOG) (specialists), competent in laparoscopic surgery such as laparoscopic ovarian cystectomy without complexity, laparoscopically assisted vaginal hysterectomy (uterine artery taken vaginally) without complexity and excision of stage 2 endometriosis, oophorectomy or removal of an ectopic pregnancy and willing to complete all required training days in full, as well as the necessary study assessment and reporting. At each hospital, up to three surgeons will be selected for training.

Other surgeons not selected for training will be asked to also enter data on all their hysterectomies as comparison group.

Theatre staff, hospital administrative staff and other relevant hospital participants are eligible if they are involved in the training programme and willing to participate in semistructured interviews to assess their personal views on benefits and future improvements of the training programme.

\section{Recruitment}

Potentially eligible hospitals known to offer hysterectomies will be contacted by the project manager to assess historical (3-year) hysterectomy activity (TAH, TLH, other), to explore the need for TLH training, and interest in participating in the trial.

\section{The TLH procedure}

For consistency, throughout all training and coaching, the surgical mentors perform the TLH procedure according to the steps described by McCartney and Obermair ${ }^{13}$; it is expected that the surgical trainees also become skilled in performing the operation according to those steps.

\section{Development and piloting of the training model}

The training model was developed and refined through a pilot feasibility study at one hospital, involving one trainer (with experience of $>2000$ TLHs) and three qualified gynaecological surgeons as trainee participants. The surgical trainee participants were Fellows of RANZCOG and already possessed general laparoscopic skills, but had minimal practical experience with TLH.

The model comprises three sequential phases: (1) Planning and preparation; (2) Delivery of surgical training and (3) Programme evaluation (figure 2).

\section{Study procedures}

Phase 1: planning and preparation

The first phase of the training model involves selection of the training-hospital, using the hospital selection tool questionnaire; identification of appropriate surgical mentors, using the surgical trainer/mentor selection tool questionnaire; and selection of appropriate surgical trainees (table 1).

All SMs attend a TLH train-the-trainer course; this practical course teaches surgical coaching skills and aims to equip the attendees as trainers. The course was adapted from existing train-the-trainers courses in flexible endoscopy and laparoscopic colorectal surgery. ${ }^{31} 32$

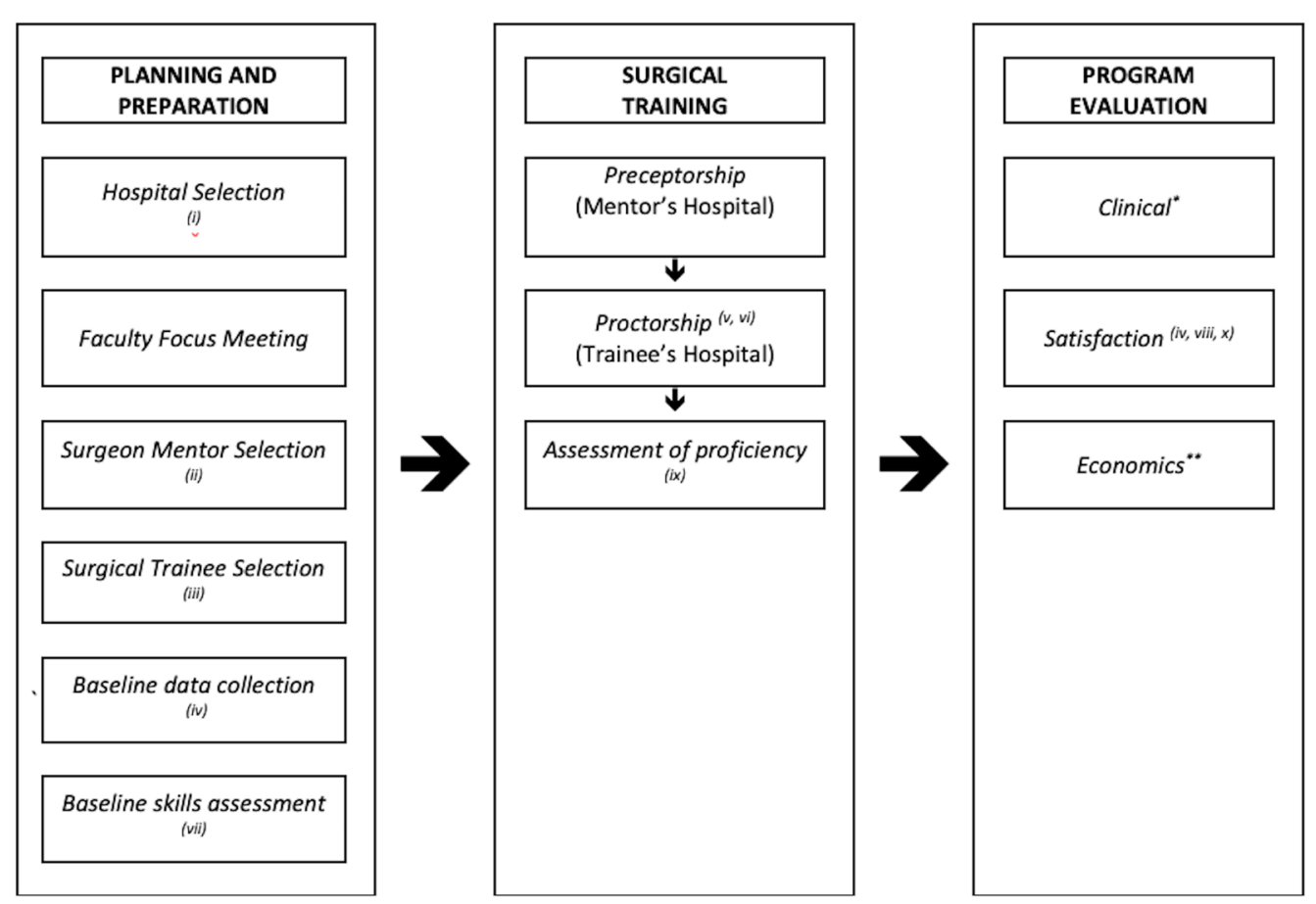

Figure 2 The Implementation of Minimally Invasive Hysterectomy Trial (IMAGINE) model for total laparoscopic hysterectomy training and mentoring. Roman numeral superscripts indicate references to the relevant assessment tool contained in the appendix. *, data from SurgicalPerformance; ${ }^{* \star}$, Medicare Benefit Schedule items. 
To maintain surgical volume for the trainees, a maximum of three STs may be trained simultaneously, the remaining surgeons not selected for TLH training support their colleagues in the training, and offer their hysterectomy cases to ensure that sufficient training cases are available; in return, non-selected surgeons are offered the same benefits to them in the future. Once these steps have been completed, a faculty focus meeting is held at the training hospital, during which information on surgical case load and key outcomes such as conversions at baseline are reviewed, and organisational/surgical trainee barriers to the adoption of TLH are discussed. This includes important practical concerns such as providing sufficient training cases to the STs, having a dedicated theatre that would be exempt from surgical target pressures, an anaesthetist that would support a reversed Trendelenburg position for laparoscopic surgery and all necessary equipment available to make the training feasible. To ensure that nursing staff, as well as the STs, gain experience and expertise, the SM, department head and the nursing theatre managers identify two scrub nurses to be involved throughout the training process. Attendance is also offered to anaesthetic staff.

\section{Phase 2: delivery of surgical training}

Surgical training is delivered through a sequential process of preceptorship (conducted at the SM's hospital), proctorship (conducted at the STs' hospital) and assessment and proficiency certification. Each trainee receives a laparoscopic simulator training device for personal use, which allows practising laparoscopic techniques in between training sessions.

\section{Training case selection}

For both preceptorship and proctorship stages, suitable patients are selected in advance by the SM in consultation with the trainees. Criteria are: (1) suitable patient classified as low risk, as measured by the SurgicalPerformance Risk of Surgical Complications app (RISC) ${ }^{33}$; (2) uterus size <10 weeks; (3) no previous laparotomy; (4) $\leq 2$ previous caesarean sections; (5) a reasonably mobile uterus; and (6) not being on blood thinning medication.

\section{Preceptorship}

Preceptorship aims to demonstrate the flow of TLH in a well-performing team and surgical environment, it shows visual aspects of the procedure and surgical setup, the atmosphere in theatre and the sound levels that the STs should aim for when proficient.

Preceptorship is provided in two stages: stage 1 involves a 1-day workshop attended by three STs and two local surgical scrub nurses. The topics comprise composition of a surgical team, surgical setup, positioning of equipment, primary and secondary port placement, surgeon's posture, effective use of laparoscopic instruments and an overview of the steps of the TLH procedure. During this stage, the SM also explains study procedures, ensures baseline surveys are completed, and explains how to use the database for recording AEs. Stage 2 involves the STs and the nursing staff attending a live TLH. The SM acts as the lead surgeon, and through demonstration reinforces the topics covered in stage 1 of preceptorship while introducing the team to each practical step of the TLH. Each surgery is video-recorded and used to facilitate a postsurgery debrief discussion between the SM and the team.

\section{Proctorship}

Proctored training consists of up to 10 training days conducted by the SM, with up to three TLH procedures conducted per day; it is provided in a dedicated training theatre at the STs' hospital using an identical configuration to that used in the preceptorship stages. The SM introduces themselves to everyone in the operating theatre, explains the aim of the day's session and then checks the equipment. The patient is brought into the operating room, the SM supervises the patient positioning, discusses equipment, setup and port placement. The SM and STs agree on a set of stopping rules-if/when a member of the surgical team calls for a stop, the operation will be paused. A stop is typically called to pause and provide an opportunity to explain anatomy or a surgical procedural step. Not adhering to the stop may translate into a lost opportunity for learning. A stop call does not necessarily translate into the operation being taken over by the SM.

During proctorship, the SM acts as the primary surgeon for the first case, with the STs acting as surgical assistants. For subsequent cases, the SM acts as surgical assistant; to avoid fatigue and exhaustion, for the first two training days, the STs perform only part of the procedure. As needed, the SM takes over to demonstrate specific procedural steps. As the STs become increasingly familiar with the TLH technique, the active involvement of the SM decreases, eventually just being present in the operating room supervising and demonstrating on the screen. Proctorship is the longest and most demanding component of TLH training. One of the challenges is for the trainees to renounce previously developed habits which are incompatible with a TLH operation.

During the training period, the SM may approve that the STs could assist each other with TLHs outside of the regular proctored surgical training days, subject to them providing the mentor with updates on surgical outcomes.

As with the preceptorship stage, to allow debriefing and to illustrate learning points, a video recording of each case is made. During debriefing, the SM provides detailed feedback to the STs, and the STs provide feedback on the SMs training, ideas to enhance the experience and goals they would like to set for the next session.

Following each surgical training day, the STs are provided with tasks to complete before the next session. These tasks include reviewing videos of TLH procedures, active watching of tutorial videos and writing of affirmations on how to complete specific surgical tasks. Between training days, the surgical mentor is available to answer questions from the trainees by email. 
Formative assessments of both the STs and SM are conducted after each case including completing The Global Operative Assessment of Laparoscopic Skills tool. These assessments help the ST to identify their strengths and weaknesses, and to target areas for improvement; they also allow the SM to adapt their coaching style as needed.

\section{Assessment of proficiency}

On successful independent completion of at least 10 TLHs, or any time thereafter, STs can submit deidentified video-recordings of two completed TLHs for independent assessment by two senior laparoscopic surgeons. Assessments will be conducted using the Laparoscopic Competency Assessment Tool (L-CAT) provided in the online supplementary appendix. ${ }^{32}$

\section{Phase 3: programme evaluation}

The programme is evaluated by: examining the surgical outcomes of the patients who underwent surgery during the training programme, experiences of the STs; experiences of the SM; the theatre staff, hospital staff and other relevant stakeholders involved in the training programme. Qualitative interviews with nursing and theatre staff will allow assessment of the benefits of the training programme for their practice, and any barriers that the whole theatre team may envisage for a future rollout of training. By comparing the surgical outcomes of patients treated during the training programme with those of expert surgeons, it will be determined whether the treatment that patients received was equivalent to best practice. A summative assessment of Satisfaction with Laparoscopic Hysterectomy Training Programme is completed by each ST at the conclusion of the programme. Semistructured interviews are conducted to explore participants' knowledge attitudes, and reflections about the programme, the integration of the new surgical skills into day-to-day practice, the ability of the programme to induce change in surgical approach, views on whether the training was successful and skills will be maintained and whether the programme could be used for future training. Interviews are recorded and transcribed verbatim for thematic analysis. In parallel to collecting TAH rates, those of hysterectomies done vaginally, using a laparoscopic-assisted vaginal or TLH approach will also be collected from before intervention throughout to follow-up.

\section{Outcomes}

Primary

The proportion of hysterectomies performed by TAH comparing preintervention baseline and postintervention rates. We aim to decrease TAH by at least $30 \%$ in $75 \%$ of the trainee surgeons. Based on published evidence from other countries and based on our own experience from a pilot study, we expect that a surgical training programme to teach TLH will achieve a higher TLH rate, which will translate into better patient outcomes and reduced surgical $\mathrm{AEs}^{320}{ }^{22}$; a decrease in TAH by $30 \%$ in at least $75 \%$ of the trainees would be clinically important.

\section{Secondary}

1. Number of hospitals screened, eligible, agree to commit to the training programme, number of hospitals that complete the training programme.

2. Number of surgeons screened for eligibility, eligible, agree and committed to the training programme, number who complete the training programme, and achieved proficiency as assessed by two independent assessors who review two deidentified videos of two independently trainee completed TLHs using the Competency Assessment Score.

3. Proportion of trainees achieving proficiency in correct theatre setup, vascular exposure, mobilisation and surgery closure as assessed by the trainer using the Formative assessment trainees' form; change in proportion proficient over time).

4. AEs (conversion from TLH to TAH, any anaesthetic incident, intraoperative visceral injury, red cell transfusions, hospital stay greater than 7 days, incidental finding of a malignancy, unplanned readmission, intensive care unit (ICU) admission or return to theatre, postoperative pulmonary embolus (PE) or deep vein thrombosis (DVT), development of a fistula, vault haematoma, vaginal vault dehiscence or pelvic infection).

5. Hospital LoS (days).

6. Cost-effectiveness (cost items: theatre staffing costs; equipment and consumables; Medicare Benefits Schedule items for surgical and anaesthetics fees; costs of health services used after surgery; costs of bed days; and costs due to AEs, readmissions or visits to the emergency department).

7. Trainee surgeon proficiency with TLH assessed by two independent assesses from two trainee submitted anonymised videos (L-CAT Competency Assessment Score).

\section{Enrolment}

The hospital is the unit of analysis. All selected STs within a hospital will be assigned to receive the training programme at the same time. Other hospitals not yet assigned to intervention will continue with standard care until they are ready to start intervention. Hospitals will be informed of their intervention start 1 month prior to commencement. Blinding of hospitals/STs is not feasible. If several hospitals are ready to receive the intervention at the same time, random switching allocation will be performed by the National Health and Medical Research Council (NHMRC) Clinical Trials Centre using a computerised system. Hospitals must demonstrate eligibility and hospital site-specific approvals before commencement.

\section{Duration}

The study commenced on 3 August 2017 and is expected to require a maximum of 36 months to complete (figure 1) with hospitals entering the training phase sequentially. 
This comprises approximately 3 months setup, $\geq 3$ months of baseline data collection, 24 months intervention, $\geq 3$ months of follow-up data collection and approximately 3 months for analysis.

\section{Data collection and management}

The study manager will record results of the following assessments listed in figure 2 in Research Electronic Data Capture $^{34}$ : (2) hospital selection tool; (2) trainer/mentor selection tool; (3) surgical training programme participant selection tool; (4) questions for O\&G surgeons; (5) formative assessment of the trainees; (6) formative assessment of the trainer/mentor; (7) the Global Operative Assessment of Laparoscopic Skills; (8) satisfaction with laparoscopic hysterectomy training programme; (9) L-CAT tool; and (10) Medicare Benefits Schedule codes. Clinical outcomes data will be collected using the SurgicalPerformance surgical reflection tool. ${ }^{35}$ STs or their representatives will enter information on patient information, surgical procedure details and outcomes directly into their account at SurgicalPerformance.com or provide their data to be entered by trial staff on their behalf. Similarly, data will also be entered by the surgeons who were not selected for training to allow comparison.

Data collected from participants will remain confidential at all times. No identifiable data will ever be shared with third parties. All questionnaires, screening tools, interview recordings and transcripts, videos and other data will only have the participant ID number on them to protect privacy. The study ID numbers will be password-protected and only accessible to study investigators and project manager. Electronic study materials will be held in password-protected computers and hard copy documents will be stored in secure cabinets. All data transferred to and from SurgicalPerformance are encrypted.

\section{Statistical analysis}

Primary outcome

The primary outcome is the proportion of hysterectomies performed by the trainees by TAH preintervention baseline and postintervention. For the programme to be worthwhile, we wish to decrease TAH by $30 \%$ in $75 \%$ of the trainees. We assume that a higher TLH rate will translate into better patient health outcomes including surgical $\mathrm{AEs}^{30}{ }^{36}$; a drop in TAH by $30 \%$ is clinically relevant. The primary outcome of the proportion of TAH procedures will be analysed within each site using $\chi^{2}$ test. These proportions will then be pooled (using inverse variance weighting) across the hospital sites to provide an overall difference in the rate of TAH for the participating trainees.

\section{Secondary outcomes}

Secondary outcomes will be increase in surgeon surgical skills, as assessed by the formative assessment forms during training; proficiency in TLH (laparoscopic assessment tool)-independently assessed by expert reviewers from two videos submitted by the trainees at the end of the training programme; AEs and hospital LoS throughout the study period. Data on surgical approach, conversion from TAH to TLH, any anaesthetic incident, intraoperative visceral injury, red cell transfusions, hospital stay $>7$ days, incidental finding of malignancy, unplanned readmission, ICU or return to theatre, postoperative PE or DVT, development of fistula, vault haematoma, vaginal vault dehiscence or pelvic infection will be extracted from the database. These will contribute to a weighted composite complication score. We aim for a $20 \%$ reduction in surgical complications. Data from non-training surgeons will be used to measure changes over time in surgical practice without intervention. Economic calculations will be undertaken estimating the costs of providing the training programme, costs of AEs and hospital bed days, comparing each hospital from pre to post training, and of surgery conducted by trainee surgeons compared with other surgeons not yet in the training programme. The satisfaction with the training programme rating scales will be summarised to provide a mean score (SD) for each of the sections related to the trainer/mentor, the hospital/peer support, overall training, training objectives as well a summary score overall. To assess the unadjusted and adjusted strength of association between participants' satisfaction with the programme, and trainer, trainee or hospital characteristics linear, logistic or generalised linear regression models will be fitted, depending on the distribution of the outcome variable.

\section{Qualitative analysis}

All interviews will be transcribed verbatim. Transcripts will be read and re-read by two independent researchers. The semistructured interview questions will be used as a priori codes and additional codes will be developed using deductive content analysis. Using a framework approach themes will then be extracted, and compared between the two readers. Discrepancies will be discussed with other members of the research team until resolved. Data will be presented with representative direct quotes.

\section{Participant and public involvement}

Before planning the IMAGINE trial, we conducted interviews with 10 women who had had a hysterectomy in the past, and then surveyed over 2600 women, which helped inform the rationale for the study. Each year, we hold a patient forum to inform women about the ongoing research conducted by the Queensland Centre for Gynaecological Cancer. We also provide updated written summaries on our public facing website, and send biannual newsletters to patients and interested members of the public.

\section{Monitoring and quality assurance \\ Process quality}

A study manager has overall responsibility for monitoring compliance with the study protocol and for initiating any remedy requests. All SMs and STs are provided with a copy of the protocol and a training manual which 
describes the practical steps necessary to implement the programme and the standard operating procedures.

\section{Data quality}

On a monthly basis, the study manager will assess: (2) completeness and timeliness of study data collectioncompleteness of information in the online Surgical Performance (SP) database is determined by comparing it with extracts from the operating room management information system); (ii) study data validity - a comparison of SP versus the medical record is made for a random sample of data $(20 \%$ for each training centre, $5 \%$ during baseline, $10 \%$ during intervention and $5 \%$ during follow-up) with a target of $<5 \%$ inconsistency between sources. Where problems of completeness or inconsistency are identified, then the study manager will request the participants to remedy.

\section{Ethics and dissemination}

The study is registered NCT03617354.

\section{DISCUSSION}

The IMAGINE trial is innovative; it will formally test a structured training model intended to develop advanced laparoscopic competency in gynaecological surgeons, so that they may complete a hysterectomy entirely laparoscopically.

Hysterectomy is the most common major gynaecological surgical procedure (almost 30000 cases/year in Australia). ${ }^{1}$ While high-level evidence is available to suggest that compared with less invasive approaches, TAH is associated with inferior health outcomes, 311 15-20 22 a significant proportion of women still receive TAH. It has been proposed that increasing the adoption of TLH would reduce the rate of TAH and subsequently improve surgical outcomes as well as provide significant cost savings for funders of healthcare. ${ }^{70}$

In previous work, we interviewed and surveyed Australian gynaecologists. ${ }^{27}$ It is apparent that Australian gynaecologists prefer to offer the hysterectomy through a vaginal approach; however, if a vaginal approach is infeasible, many resort to an open, abdominal surgical approach. Only a minority of Australian gynaecological surgeons considered themselves sufficiently trained to offer patients a TLH, and this lack of training is the main impediment to them offering TLH as an alternative to TAH. We also surveyed women who had a hysterectomy, as active querying by patients to received TLH may contribute to a change in hysterectomy approach used. However, we found that women mainly knew about TAH, tended to follow the recommendation by their doctor and rarely sought a second opinion. Therefore, patient pressure was unlikely to facilitate a quicker move to TLH, and providing gynaecological surgeons with training to allow them to confidently offer TLH to their patients was the most promising strategy.
Information is abundant on the assessment of surgical skills in specialist trainees and specialists, ${ }^{37} 38$ quantifying the surgical skills required for laparoscopic hysterectomy ${ }^{39} 40$ and documenting improved surgical skills from baseline through training. The UK programme on laparoscopic colorectal cancer surgery has reported extensively about the key elements and outcomes of the training programme. ${ }^{41-43}$ However, comparatively little information is available in the literature about how surgical training processes for practising consultant gynaecological surgeons may be established at an organisational level. The research study described here aims to close that evidence gap.

\section{CONCLUSION}

If successful, this training model will equip surgeons with the skills to offer a minimally invasive approach to hysterectomy which will translate into better care for women, while reducing health system costs. The findings may be useful in informing a scaled-up model for laparoscopic gynaecological surgical training.

\section{Author affiliations}

${ }^{1}$ Centre for Clinical Research, University of Queensland, Brisbane, Queensland, Australia

${ }^{2}$ Queensland Centre for Gynaecological Cancer, Royal Brisbane and Women's Hospital, Herston, Queensland, Australia

${ }^{3} \mathrm{HBI}, \mathrm{QUT}$, Brisbane, Queensland, Australia

${ }^{4}$ CTC, University of Sydney, Sydney, New South Wales, Australia

${ }^{5}$ Department of Surgery \& Cancer, Faculty of Medicine, Imperial College, London, UK ${ }^{6}$ Department of Surgery, University Hospitals Plymouth NHS Trust, Derriford Hospital, Plymouth, UK

${ }^{7}$ Centre of Health Services Research, Faculty of Medicine, University of Queensland, Brisbane, Queensland, Australia

${ }^{8}$ School of Public Health and Social Work, Institute for Health and Biomedical Innovation, Queensland University of Technology, Brisbane, Queensland, Australia

Contributors A0, GBH, MGC and MJ conceived the study; A0, MJ, NG and VG designed the study; $V G$ developed the statistical methods and is the study statistician. NG is the study health economist who will assess the relative effectiveness of the intervention. NRA contributed to the design and drafted the initial manuscript. AH is the study manager and continued to development of the protocol. All authors contributed to critical review and revision of the manuscript and approved the final version.

Funding This work is supported by NHMRC Partnership Project for Better Health, grant (1145961); Monika Janda is supported by an NHMRC Translating Research into Practice Fellowship (1151021). The work has received funding from Medtronic as an industry partner, and seed funding from Metro North Hospital and Health Service.

Disclaimer The funders did not have any role in the conduct of the study or reporting of outcomes.

Competing interests None declared.

Patient consent for publication Not required.

Ethics approval The study has been approved by the Royal Brisbane and Women's Hospital Human Research Ethics Committee (Approval HREC/16/QRBW/564), as well as received site-specific approval by all participating hospitals. The initial pilot study was assessed by the Metro South Hospital and Health Service Human Research Ethics Committee (reference HREC/16/QPAH/468) and was deemed a quality assurance activity and exempt from the requirement for full ethical review and approval.

Provenance and peer review Not commissioned; externally peer reviewed. 
Open access This is an open access article distributed in accordance with the Creative Commons Attribution Non Commercial (CC BY-NC 4.0) license, which permits others to distribute, remix, adapt, build upon this work non-commercially, and license their derivative works on different terms, provided the original work is properly cited, appropriate credit is given, any changes made indicated, and the use is non-commercial. See: http://creativecommons.org/licenses/by-nc/4.0/.

\section{REFERENCES}

1. Australian Institute of Health and Welfare (AlHW). National Hospital Morbidity Database. Procedures and healthcare interventions 2013-14. Canberra, Australia, 2016.

2. European Union. Eurostat - Surgical operations and procedure statistics. 2018.

3. Aarts JWM, Nieboer TE, Johnson N, et al. Surgical approach to hysterectomy for benign gynaecological disease. Cochrane Database Syst Rev 2015;194.

4. Nieboer TE, Hendriks JC, Bongers MY, et al. Quality of life after laparoscopic and abdominal hysterectomy: a randomized controlled trial. Obstet Gynecol 2012;119:85-91.

5. Nieboer TE, Spaanderman ME, Bongers MY, et al. Gynaecologists estimate and experience laparoscopic hysterectomy as more difficult compared with abdominal hysterectomy. Gynecol Surg 2010;7:359-63.

6. Siew TY. Laparoscopy Versus Laparotomy for the Management of Early-Stage Endometrial Cancer. Cancer Nurs 2014;37:2-3.

7. Graves N, Janda M, Merollini K, et al. The cost-effectiveness of total laparoscopic hysterectomy compared to total abdominal hysterectomy for the treatment of early stage endometrial cancer. BMJ Open 2013;3:e001884.

8. Kluivers KB, Johnson NP, Chien P, et al. Comparison of laparoscopic and abdominal hysterectomy in terms of quality of life: a systematic review. Eur J Obstet Gynecol Reprod Biol 2008;136:3-8.

9. Korolija D, Sauerland S, Wood-Dauphinée S, et al. Evaluation of quality of life after laparoscopic surgery: evidence-based guidelines of the European Association for Endoscopic Surgery. Surg Endosc 2004;18:879-97.

10. Lumsden MA, Twaddle S, Hawthorn R, et al. A randomised comparison and economic evaluation of laparoscopicassisted hysterectomy and abdominal hysterectomy. BJOG 2000;107:1386-91.

11. Walsh CA, Walsh SR, Tang TY, et al. Total abdominal hysterectomy versus total laparoscopic hysterectomy for benign disease: a metaanalysis. Eur J Obstet Gynecol Reprod Biol 2009;144:3-7.

12. Medtronic. Global Value Dossier for minimally invasive surgery: Hysterectomy. 2016. http://www.medtronic.com/content/dam/ covidien/library/us/en/clinical-procedural-therapy/gynecology/ hysterectomy-global-value-dossier.pdf

13. McCartney AJ, Obermair A. Total laparoscopic hysterectomy with a transvaginal tube. J Am Assoc Gynecol Laparosc 2004;11:79-82.

14. Obermair A, Ginbey P, McCartney AJ. Feasibility and safety of total laparoscopic radical hysterectomy. J Am Assoc Gynecol Laparosc 2003;10:345-9.

15. Goolab BD. Vaginal hysterectomy and relative merits over abdominal and laparoscopically assisted hysterectomy. Best Pract Res Clin Obstet Gynaecol 2013;27:393-413.

16. Gupta S, Manyonda I. Hysterectomy for benign gynaecological disease. Obstet Gynaecol Reprod Med 2014;24:135-40.

17. Mäkinen J, Brummer T, Jalkanen J, et al. Ten years of progressimproved hysterectomy outcomes in Finland 1996-2006: a longitudinal observation study. BMJ Open 2013;3:e003169.

18. Wright JD, Ananth CV, Lewin SN, et al. Robotically assisted vs laparoscopic hysterectomy among women with benign gynecologic disease. JAMA 2013;309:689-98

19. Fader AN, Weise RM, Sinno AK, et al. Utilization of minimally invasive surgery in endometrial cancer care: a quality and cost disparity. Obstet Gynecol 2016;127:91-100.

20. Janda M, Gebski V, Brand A, et al. Quality of life after total laparoscopic hysterectomy versus total abdominal hysterectomy for stage I endometrial cancer (LACE): a randomised trial. Lancet Oncol 2010;11:772-80.
21. Janda M, Gebski V, Davies LC, et al. Effect of total laparoscopic hysterectomy vs total abdominal hysterectomy on diseasefree survival among women with stage I endometrial cancer: a randomized clinical trial. JAMA 2017;317:1224-33.

22. Obermair $\mathrm{A}$, Janda $\mathrm{M}$, Baker $\mathrm{J}$, et al. Improved surgical safety after laparoscopic compared to open surgery for apparent early stage endometrial cancer: results from a randomised controlled trial. Eur $J$ Cancer 2012;48:1147-53.

23. El Hachem L, Andikyan V, Mathews S, et al. Robotic single-site and conventional laparoscopic surgery in gynecology: clinical outcomes and cost analysis of a matched case-control study. $J$ Minim Invasive Gynecol 2016;23:760-8.

24. Ind $\mathrm{T}$, Laios $\mathrm{A}$, Hacking $\mathrm{M}$, et al. $\mathrm{A}$ comparison of operative outcomes between standard and robotic laparoscopic surgery for endometrial cancer: A systematic review and meta-analysis. Int J Med Robot Comp 2017;13:e1851.

25. Vuorinen R-LK, Mäenpää MM, Nieminen K, et al. Costs of RoboticAssisted Versus Traditional Laparoscopy in Endometrial Cancer. Int J Med Robot 2017;27:1788-93.

26. Wright JD, Ananth CV, Tergas Al, et al. An Economic Analysis of Robotically Assisted Hysterectomy. Obstet Gynecol 2014;123:1038-48.

27. Janda M, Armfield NR, Kerr G, et al. Surgical approach to hysterectomy and barriers to using minimally invasive methods. Aust N Z J Obstet Gynaecol 2018;58:690-5.

28. Janda M, Armfield NR, Page K, et al. Factors influencing women's decision making in hysterectomy. Patient Educ Couns 2018;101:504-10.

29. Jokinen E, Brummer T, Jalkanen J, et al. Hysterectomies in Finland in 1990-2012: comparison of outcomes between trainees and specialists. Acta Obstet Gynecol Scand 2015;94:701-7.

30. Gale J, Cameron C, Chen I, et al. Increasing Minimally Invasive Hysterectomy: A Canadian Academic Health Centre Experience. $J$ Obstet Gynaecol Can 2016;38:141-6.

31. LapCo International. 2018. Available: http://lapcointernational.co.uk/

32. Coleman MG, Cecil T. Laparoscopic Colorectal Surgery: The Lapco Manual: CRC Press, 2017.

33. SurgicalPerformance. RISC: SurgicalPerformance App. 2018. https:// surgicalperformance.com/news-media/risc-surgicalperformanceapp/

34. Harris PA, Taylor R, Thielke R, et al. Research electronic data capture (REDCap)-A metadata-driven methodology and workflow process for providing translational research informatics support. J Biomed Inform 2009;42:377-81.

35. Surgical Performance, 2018. Available: https://surgicalperformance. com/about/

36. Makinen J, et al. Morbidity of 10110 hysterectomies by type of approach. Hum Reprod 2001;16:1473-8.

37. Szasz P, Louridas M, Harris KA, et al. Assessing technical competence in surgical trainees: a systematic review. Ann Surg 2015;261:1046-55

38. van Hove PD, Tuijthof GJM, Verdaasdonk EGG, et al. Objective assessment of technical surgical skills. Br J Surg 2010;97:972-87.

39. Einarsson JI, Suzuki Y. Total laparoscopic hysterectomy: 10 steps toward a successful procedure. Rev Obstet Gynecol 2009;2:57.

40. Tremblay C, Grantcharov T, Urquia ML, et al. Assessment tool for total laparoscopic hysterectomy: a Delphi consensus survey among international experts. J Obstet Gynaecol Can 2014;36:1014-23.

41. Coleman MG, Hanna GB, Kennedy R, et al. The National Training Programme for laparoscopic colorectal surgery in England: a new training paradigm. Colorectal Disease 2011;13:614-6.

42. Mackenzie H, Cuming T, Miskovic D, et al. Design, delivery, and validation of a trainer curriculum for the National laparoscopic colorectal training program in England. Ann Surg 2015;261:149-56.

43. Miskovic D, Ni M, Wyles SM, et al. Is competency assessment at the specialist level achievable? A study for the national training programme in laparoscopic colorectal surgery in England. Ann Surg 2013;257:476-82.

44. The Royal Australian and new Zealand College of Obstericians and Gynaecologists (RANZCOG). Guidelines for performing gynaecological endoscopic procedures, 2018.

45. Vassiliou MC, Feldman LS, Andrew CG, et al. A global assessment tool for evaluation of intraoperative laparoscopic skills. Am J Surg 2005; 190:107-13. 